\title{
Mesothelioma environment comprises cytokines and T-regulatory cells that suppress immune responses
}

\author{
J.P.J.J. Hegmans*, A. Hemmes*, H. Hammad*, L. Boon" \\ H.C. Hoogsteden* and B.N. Lambrecht*
}

ABSTRACT: Malignant mesothelioma is a cancer with dismal prognosis. The objective of the present study was to address the role of the immune system, tumour micro-environment and potential immunosuppression in mesothelioma.

Expression profiles of $\mathbf{8 0}$ cytokines were determined in the supernatant of mesothelioma cell lines and the original patient's pleural effusion. Influx of immune effector cells was detected by immunohistochemistry.

Angiogenin, vascular endothelial growth factor, transforming growth factor- $\beta$, epithelial neutrophil-activating protein-78 and several other proteins involved in immune suppression, angiogenesis and plasma extravasation could be detected in both supernatant and pleural effusion. Surrounding stroma and/or infiltrating cells were the most likely source of hepatocyte growth factor, macrophage inflammatory protein (MIP)-1 $\delta$, MIP-3 $\alpha$, neutrophil-activating peptide2 , and pulmonary and activation-regulated chemokine that can cause leukocyte infiltration and activation. There was a massive influx of CD4+ and CD8+ T-lymphocytes and macrophages, but not of dendritic cells, in human mesothelioma biopsies. It was further demonstrated that human mesothelioma tissue contained significant amounts of Foxp3+CD4+CD25+ regulatory T-cells. When these CD25+ regulatory T-cells were depleted in an in vivo mouse model, survival increased.

Mesothelioma is infiltrated by immune effector cells but also contains cytokines and regulatory T-cells that suppress an efficient immune response. Immunotherapy of mesothelioma might be more effective when combined with drugs that eliminate or control regulatory T-cells.

\section{KEYWORDS: Cancer, Foxp3, immunosuppression, immunotherapy, micro-environment}

$\mathbf{M}$ alignant mesothelioma (MM) is a highly aggressive neoplasm most often seen in patients with a history of asbestos exposure. There is a latency period of 20-40 yrs between the exposure to asbestos fibres and the first symptoms of disease. With median survival durations of 9-12 months from onset of symptoms, the prognosis is poor. To date, there is no standard curative therapy for MM. Combined modality approaches, such as extrapleural pneumonectomy followed by radiochemotherapy, result in high local recurrence rates and questionable survival benefit [1]. As MM is a weak immunogenic tumour, various groups have attempted to perform immunotherapy using cytokines or adjuvants to boost tumour immunity, with varying success [1]. In a previous study, the present authors evaluated the therapeutic efficacy of tumour lysate-loaded antigenpresenting dendritic cells (DCs) given before and/or after an i.p. tumour challenge with the mouse mesothelioma cell line AB1. DCs pulsed with tumour lysate or exosomes were effective in inducing protective cytotoxic CD8 T-cell responses and increasing survival, even when given after tumour implantation [2]. In these studies, DC treatment had a better outcome when DCs were injected early in tumour development, indicating that tumour load played an important role in survival. Although the exact sequence of events in mesothelioma induction and progression is still unknown, a range of defects that develop both inside and outside the mesothelial cell could be involved in the escape of the tumour from immune destruction. According to the immune surveillance theory, large tumours escape immune recognition by downregulating major histocompatibility complex (MHC) class I or by altering expression of tumour antigens, thus leading to an escape from cytotoxic killing

\section{AFFILIATIONS}

*Dept of Pulmonary Medicine, Erasmus MC, Rotterdam, and \#Bioceros B.V., Utrecht, The Netherlands.

CORRESPONDENCE

J.P.J.J. Hegmans

H-Ee2253a

Dept of Pulmonary Medicine

Erasmus MC

P.O. Box 1738

$3000 \mathrm{DR}$

Rotterdam

The Netherlands

Fax: 31104089453

E-mail: j.hegmans@erasmus.nl

Received:

November 162005

Accepted after revision:

February 222006

\section{SUPPORT STATEMENT}

This study was financially supported by Stichting Asbestkanker (Rotterdam, the Netherlands) and Mesothelioma Applied Research Foundation (MARF; Santa Barbara, CA, USA).

Online ISSN 1399-3003 
by CD8 cells [3]. This theory has recently been challenged, as spontaneously arising tumours in mice remain immunogenic and, instead, escape immune recognition by inducing anergy in tumour-infiltrating lymphocytes [4] or by attracting regulatory T-cells (Treg) that suppress anti-tumoural responses. It is now well established that certain tumours and the surrounding stroma generate an immunosuppressive microenvironment to suppress the effector arm of the anti-tumoural immune response (cytotoxic T-lymphocyte response inside the tumour) and the inductive arm of the immune response, i.e. the potential of antigen-presenting DCs to induce cytotoxic Tlymphocyte responses.

The present study takes an unbiased approach, using a proteomics platform and determining the presence of an array of 80 cytokines and chemokines in mesothelioma cell lines and pleural fluids of the original patients from whom the cell lines were generated. This allowed the authors to study which factors were tumour derived and which were derived from infiltrating immune cells or surrounding stroma. Tumours were heavily infiltrated with CD4+ and CD8+ T-cells and macrophages, but DCs were strikingly absent. Surprisingly, Foxp3+ CD25+ Treg, which were previously shown to promote tumour progression in other cancer models, were found. Removal of these cells led to increased survival in a transplantable mouse model of mesothelioma. These findings suggest there are multiple levels by which MM escapes immune recognition.

\section{METHODS}

\section{Patient material and preparation of cell lines for analysis}

After obtaining informed consent, pleural fluid was collected from histologically proven mesothelioma patients $(n=6)$ who presented with large pleural effusions. In most cases, indication for pleural fluid evacuation was exertional dyspnoea relief. All patients were 67-88-yr-old males who were treated with best supportive care. Thoracocentesis was performed using fine-needle aspiration inserted into the pleural cavity and collected in sterile tubes without anticoagulant. Pleural cells were removed from pleural effusions using centrifugation at $3,000 \times g$ for $20 \mathrm{~min}$ at $4^{\circ} \mathrm{C}$, and the supernatant was stored in aliquots at $-80^{\circ} \mathrm{C}$. Four long-term mesothelioma cell lines were generated from these pleural effusions and extensively characterised as described earlier [5]. This included determining the cellular DNA content, immunohistochemistry, tumourigenicity in vivo, virus and/or bacterial contamination, karyotyping, and human leukocyte antigen typing. Cell lines were kept in long-term cell culture ( $>50$ passages) before using for supernatant testing. Pleural fluid from these patients was still available for analysis.

In a separate group of patients $(n=4)$, tumour biopsies were obtained through medical thoracoscopy and processed for immunohistology.

\section{Proteomics platform to analyse cytokines and chemokines}

An antibody-based cytokine array system (RayBiotech, Inc., Norcross, GA, USA) was used to determine cytokine expression profiles in supernatant of mesothelioma cell lines $(n=4)$ and the corresponding patient's pleural effusions $(n=6)$. Cell lines were grown in T175 culture flasks to $80 \%$ confluence. Medium was then replaced by $12-\mathrm{mL}$ RPMI containing $1 \%$ foetal calf serum and incubated at $37^{\circ} \mathrm{C}$. The following day, supernatant was collected and centrifuged for $20 \mathrm{~min}$ at $3,000 \times g$ to remove the cells. Samples were concentrated using Centricon YM3 columns (Millipore, Billerica, MA, USA). As a negative control, $12 \mathrm{~mL}$ of the previously mentioned medium was prepared in the same way as the cell supernatant. Of these concentrated samples, $1 \mathrm{~mL}$ were applied to the RayBio membranes (human cytokine array $\mathrm{V}$; table 1). The detection was performed according the manufacturer's protocol. Quantification of cytokine expression was performed in duplicate by two independent observers (J.P.J.J. Hegmans and A. Hemmes) using a standard scale of six spots with increasing density (score 0 to 5; landing lights (positive controls) were scored as 4).

\section{Immunohistology on tumour biopsies}

Tumour biopsies were taken using medical thoracoscopy and embedded in Tissue-Tek II optimum cutting temperature medium (Miles, Naperville, IL, USA), snap-frozen and stored at $-80^{\circ} \mathrm{C}$. Tissue sections $(6 \mu \mathrm{m})$ were cut on an HM-560 cryostat (Microm, Heidelberg, Germany) and immunostaining was carried out using antibodies (table 2). Binding of antibodies was detected using the immuno-alkaline phosphatase (AP) anti-alkaline phosphatase (APAAP) method (DAKO, Glostrup, Denmark). Naphtol-AS-MX-phosphate $\left(0.30 \mathrm{mg} \cdot \mathrm{mL}^{-1}\right.$; Sigma-Aldrich Chemie B.V., Zwijndrecht, the Netherlands) and new fuchsine $\left(160 \mathrm{mg} \cdot \mathrm{mL}^{-1}\right.$ in $2 \mathrm{M} \mathrm{HCl}$; ChromaGesellschaft, Köngen, Germany) were used as substrate. The specificity of the antibodies was checked using a protein concentration-matched nonrelevant monoclonal antibody and PBS. Double staining of Foxp3 (rat immunoglobulin (Ig)G2a) and CD3 or CD25 (both mouse IgG1) was performed using AP-conjugated goat anti-rat (Sigma-Aldrich Chemie B.V.) and rat APAAP (DAKO) followed by horseradish peroxidase conjugated goat anti-mouse IgG1 (Southern Biotechnology Associates, Birmingham, AL, USA). Naphtol-AS-MX-phosphate and $1 \mathrm{mM}$ Fast Blue substrate were used as substrate for AP and NovaRed was used as substrate for horseradish peroxidise, according to the manufacturer's instructions (Vector, Burlingame, CA, USA). Alexa Fluor 647 labelled anti-human CD4 and fluorescein isothiocyanate conjugated CD25 (both BD Biosciences, San Jose, CA, USA) were used at a dilution of $1: 100$ and 1:20, respectively. Signals were captured on a Zeiss confocal laser-scanning microscope (LSM510NLO; Carl Zeiss B.V., Sliedrecht, the Netherlands).

\section{Tumour growth of murine mesothelioma after in vivo depletion of CD4+CD25+ T-cells in BALB/c mice}

Female 6-10-week-old BALB/c (H-2d) mice (Harlan, Zeist, the Netherlands) were housed under pathogen-free conditions at the animal care facility of Erasmus MC (Rotterdam, the Netherlands). Experiments were approved by the local ethical committee for animal welfare (Erasmus University Committee of Animal Experts, Rotterdam, the Netherlands) and complied with the Guidelines for the Welfare of Animals in Experimental Neoplasia by the United Kingdom Coordinating Committee on Cancer Research. The AB1 cell line, a mouse mesothelioma cell line, was kindly provided by B.W.S. Robinson (School of Medicine and Pharmacology, University of Western Australia, Sir Charles Gairdner Hospital, Perth, Australia). For in vivo depletion of CD4+CD25+ T-cells, $0.5 \mathrm{~mL}$ 
TABLE 1 The 80 cytokines probed for on the RayBio human cytokine array membranes

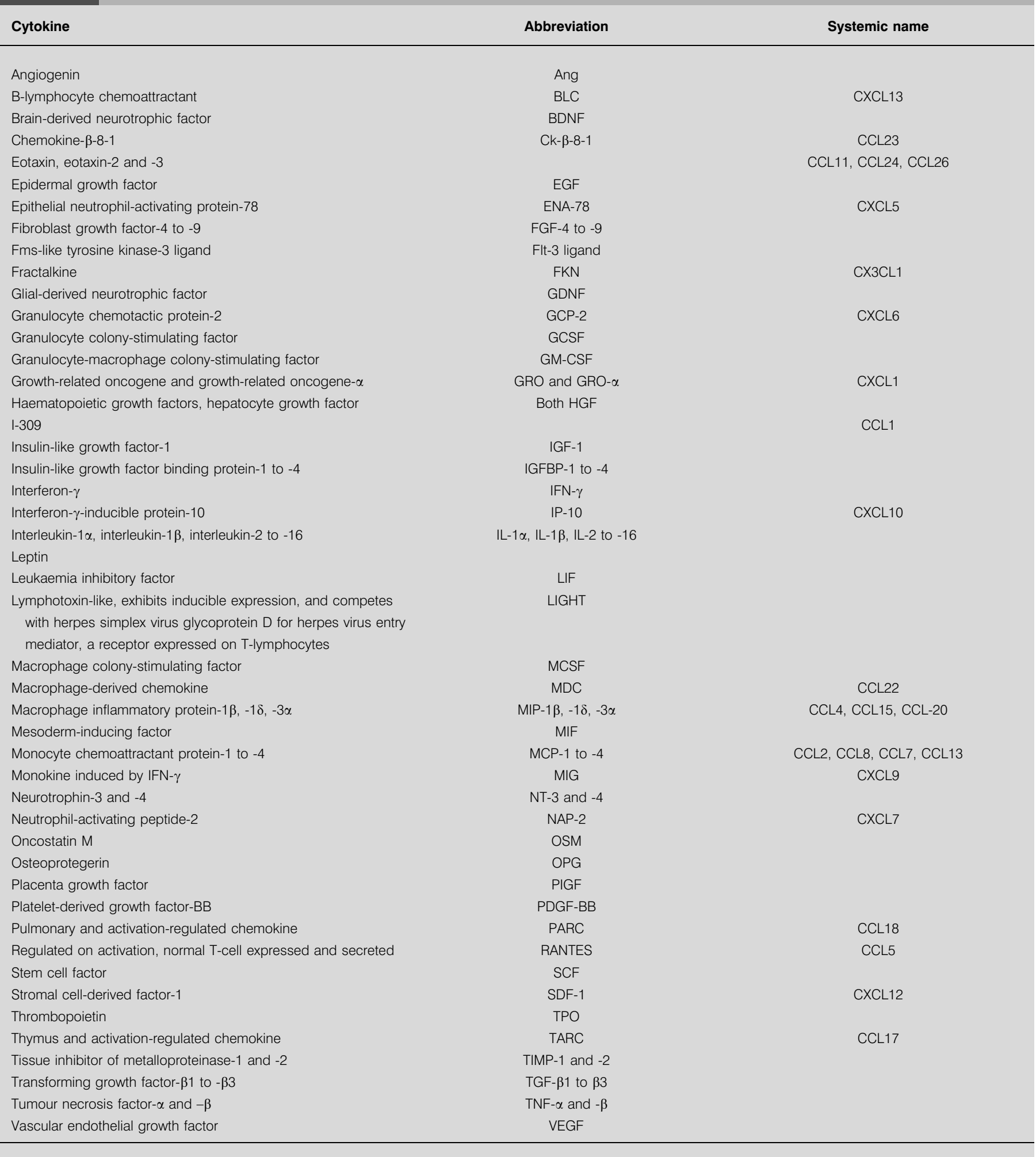

of anti-CD25 antibody (PC61) ascites fluid (kindly provided by G. Oldenhove, Université Libre de Bruxelles, Brussels, Belgium) was given intraperitoneally 25 days before tumour cell inoculation to each mouse $(n=12)$. The ascitis fluid was purified by affinity chromatography on protein A-Sepharose
4B as described by LOWENTHAL et al. [6]. As a control the authors used $0.5 \mathrm{~mL}$ of PBS $(n=12)$. On day 0 , mice were subjected to a lethal dose of $0.5 \times 10^{6} \mathrm{AB} 1$ tumour cells. The occurrence of tumour growth, body weight, physical well-being and survival were measured for 2 months, as described previously [2]. 
TABLE 2 Source and specificity of antibodies used for the immunohistochemical staining of mesothelioma sections

\begin{tabular}{|c|c|c|}
\hline BDCA2 & Plasmacytoid DCs & Miltenyi Biotech \\
\hline CD1a & DC & DAKO \\
\hline CD3 & pan-T lymphocytes & DAKO \\
\hline CD4 & T-helper/inducer lymphocytes, monocytes & DAKO \\
\hline CD14 & Monocytes (macrophages/granulocytes) & DAKO \\
\hline CD15 & Neutrophils (monocytes) & DAKO \\
\hline CD16 & NK cells, neutrophils and basophils & DAKO \\
\hline CD24 & B-cells, neutrophils, DCs & DAKO \\
\hline CD25 & Activated T-cells and, at a lower density, activated B-cells & DAKO \\
\hline Foxp3 & Regulatory T-cells & eBioscience \\
\hline HBME1 & Mesothelial cells & DAKO \\
\hline HLA DR DQ DP & MHC class II-expressing cells & DAKO \\
\hline RCK108 & Normal and malignant epithelial cells & DAKO \\
\hline $5 \mathrm{~B} 5$ & Fibroblasts & DAKO \\
\hline
\end{tabular}

DC: dendritic cell; NK: natural killer; ICAM: intercellular adhesion molecule; MHC: major histocompatibility complex. The location details of each manufacturer are as follows. Miltenyi Biotech: Bergisch Gladback, Germany; Monosan/Sanbio: Uden, the Netherlands; DAKO: Glostrup, Denmark; BD Biosciences: San Jose, CA, USA; R\&D Systems: Minneapolis, MN, USA; Chemicon: Temecula, CA, USA; eBioscience: San Diego, CA, USA.

\section{Statistical analysis}

Data are expressed as mean \pm SD. Comparisons between groups were made using the Mann-Whitney U-test for independent samples. A two-tailed p-value $<0.05$ was considered significant. Data presented as a percentage of tumour-free animals were analysed with Kaplan-Meier survival curves, using the log-rank test to determine statistical significance.

\section{RESULTS}

\section{Expression profile of pleural effusions and cell line supernatants on cytokine and chemokine array}

An antibody-based cytokine array system (RayBiotech, Inc.) was used to determine cytokine expression profiles in pleural effusion $(n=6)$ and in the supernatant of mesothelioma cell lines derived from the same patients $(n=4)$. No cytokine expression was found in the negative (medium) control. Cytokine expression levels in pleural effusions and corresponding supernatant of mesothelioma cell lines are summarised in table 3. Forty-nine cytokines were undetected in both effusion and supernatant of cultured mesothelioma cell lines. Hepatocyte growth factor (HGF), macrophage inflammatory protein (MIP)-1 $\delta$, MIP- $3 \alpha$, neutrophil-activating peptide (NAP)-2, and pulmonary and activation-regulated chemokine (PARC) were exclusively present in pleural effusions and were not detected in mesothelioma cell supernatant (fig. 1). Therefore, it is possible that these cytokines are produced and secreted in pleural effusions by stromal cells and/or inflammatory cells. Some effusions also expressed cytokine levels of eotaxin-2, interleukin (IL)-12, leptin, mesoderm-inducing factor (MIF) and oncostatin M. In contrast, supernatants of mesothelioma cell lines contain high levels of growth-related oncogene (GRO) and RANTES (regulated on activation, normal T-cell expressed and secreted). These proteins seem to be mainly secreted by tumour cells and the amount present in effusion may thus be correlated with the amount of tumour cells.

\section{Cellular recruitment in human mesothelioma tumours}

The local release of cytokines and chemotactic factors by tumour cells and surrounding stroma suggests an accumulation of leukocytic infiltrate in the vicinity of the tumour by recruitment from circulating blood cells. Immunohistochemical techniques were therefore performed to determine the inflammatory component in solid tumour tissue. Mesothelioma tumour tissue can be regarded as complex tissues, composed of localised HBME-1 positive tumour fields, separated by stroma and many CD31+ blood vessels (fig. 2). Although some patient-to-patient heterogeneity was noted, leukocyte infiltration was always detected. Macrophages (CD68) and natural killer (NK) cells (CD16) constitute the major part of the inflammatory cell infiltration. Interactions between cancer cells and host immune T-cells (pan-T (CD3), Thelper/inducer (CD4), and T-suppressor/cytotoxic lymphocytes (CD8)) were visualised inside, at the rim and in the stroma of mesothelioma specimens. However, DCs (using antibodies for Langerin, fascin, S100, CD1a, BDCA2 and CD209), eosinophils (BMK-13), mast cells (anti-chymase), B-cells (CD24), and neutrophils (CD15) were rarely detected (fig. 3). 


\begin{tabular}{|c|c|}
\hline TABLE 3 & $\begin{array}{l}\text { ted in pleural effusions and } \\
\text { othelioma cell lines }\end{array}$ \\
\hline Detected $^{\#}$ & Mean PF \pm SD:mean $\mathrm{SN} \pm \mathrm{SD}$ \\
\hline \multicolumn{2}{|l|}{$\mathrm{PF} \uparrow \uparrow$} \\
\hline HGF & $1.50 \pm 1.50: 0$ \\
\hline MIP-1 $1 \delta$ & $1.67 \pm 0.52: 0^{\star}$ \\
\hline MIP-3 $\alpha$ & $0.83 \pm 1.33: 0$ \\
\hline NAP-2 & $2.33 \pm 0.52: 0^{*}$ \\
\hline PARC & $3.50 \pm 0.55: 0^{*}$ \\
\hline \multicolumn{2}{|l|}{$\mathrm{PF} \uparrow$} \\
\hline Ang & $3.17 \pm 0.41: 2.00 \pm 0^{*}$ \\
\hline Eotaxin-2 & $0.33 \pm 0.84: 0$ \\
\hline IGFBP-1 & $1.50 \pm 1.05: 0.50 \pm 0.57$ \\
\hline IGFBP-2 & $1.83 \pm 0.98: 1.25 \pm 1.5$ \\
\hline IL-12 & $0.17 \pm 0.41: 0$ \\
\hline $\mid \mathrm{P}-10$ & $1.00 \pm 0.89: 0.75 \pm 1.50$ \\
\hline Leptin & $0.50 \pm 0.84: 0$ \\
\hline MIF & $0.67 \pm 1.04: 0.25 \pm 0.5$ \\
\hline OSM & $0.17 \pm 0.41: 0$ \\
\hline PIGF & $0.83 \pm 0.75: 0.50 \pm 1.00$ \\
\hline \multicolumn{2}{|l|}{$\mathrm{PF} \leftrightarrow \mathrm{SN}$} \\
\hline Osteoprotegrin & $0.83 \pm 0.75: 0.75 \pm 0.50$ \\
\hline VEGF & $0.67 \pm 0.82: 0.75 \pm 0.96$ \\
\hline MCP-1 & $2.33 \pm 0.51: 2.50 \pm 1.73$ \\
\hline TGF- $\beta 2$ & $0.17 \pm 0.41: 0.25 \pm 0.50$ \\
\hline ENA-78 & $0.50 \pm 1.22: 0.50 \pm 1.00$ \\
\hline TIMP-1 & $2.67 \pm 0.51: 2.50 \pm 1.29$ \\
\hline IL-6 & $4.50 \pm 0.84: 3.50 \pm 2.38$ \\
\hline MIP-1 $\beta$ & $1.00 \pm 0.63: 0.75 \pm 1.50$ \\
\hline \multicolumn{2}{|l|}{$\mathbf{S N} \uparrow$} \\
\hline BDNF & $0: 0.25 \pm 0.50$ \\
\hline IGFBP-3 & $0.33 \pm 0.51: 0.75 \pm 0.50$ \\
\hline IGFBP-4 & $0.83 \pm 0.75: 1.25 \pm 0.96$ \\
\hline TIMP-2 & $2.50 \pm 0.55: 3.25 \pm 0.50$ \\
\hline IL-8 & $3.66 \pm 1.51: 4.25 \pm 0.50$ \\
\hline \multicolumn{2}{|l|}{$\mathbf{S N} \uparrow \uparrow$} \\
\hline GRO & $1.50 \pm 0.84: 2.75 \pm 0.5^{\star}$ \\
\hline RANTES & $0.17 \pm 0.41: 1.25 \pm 0.96$ \\
\hline
\end{tabular}

For cytokine abbreviations see table 1. PF: pleural fluid; SN: cell line supernatant. $\uparrow \uparrow:$ strong increase; $\uparrow:$ moderate increase; $\leftrightarrow$ : equally expressed in PF and SN. *: cytokines that were not detected were BLC, Ck- $\beta-8-1$, EGF, eotaxin, eotaxin-3, FGF-4 to -9 Flt-3 ligand, fractalkine, GCP-2, GCSF, GDNF, GM-CSF, GRO- $\alpha, \quad$ I-309, IFN- $\gamma, \quad$ IGF- $1, \quad I L-1 \alpha,-1 \beta$ and -2 to -16 , LIF, LIGHT, MCP-2 to -4, MCSF, MDC, MIG, NT-3 and -4 , PDGF-B, SCF, SDF-1, TARC, TGF- $\beta 1$ and $-\beta 3$, TNF- $\alpha$ and $-\beta$, and TPO. ${ }^{*}: p<0.05$.

Treg can be discriminated based on the expression of CD4 and CD25, combined with the transcription factor Foxp3. Mesothelioma tissue sections were analysed by fluorescence microscopy for the phenotypic evidence of CD4+CD25+ double positive cells. T-cells were particularly detected at the rim of tumour areas CD4+CD25+ (fig. $4 \mathrm{~g}$ and $\mathrm{h}$ ). The transcription factor Foxp3, identified by SAKAGUCHI et al. [7] as a hallmark of naturally arising CD4+CD25+ Treg cells, confirmed the presence of these cells in the vicinity of the tumour (fig. 4a). Double stainings showed that the expression of Foxp3 strongly correlated with CD3 (fig. 4b) and CD25 expression (fig. 4c and d).

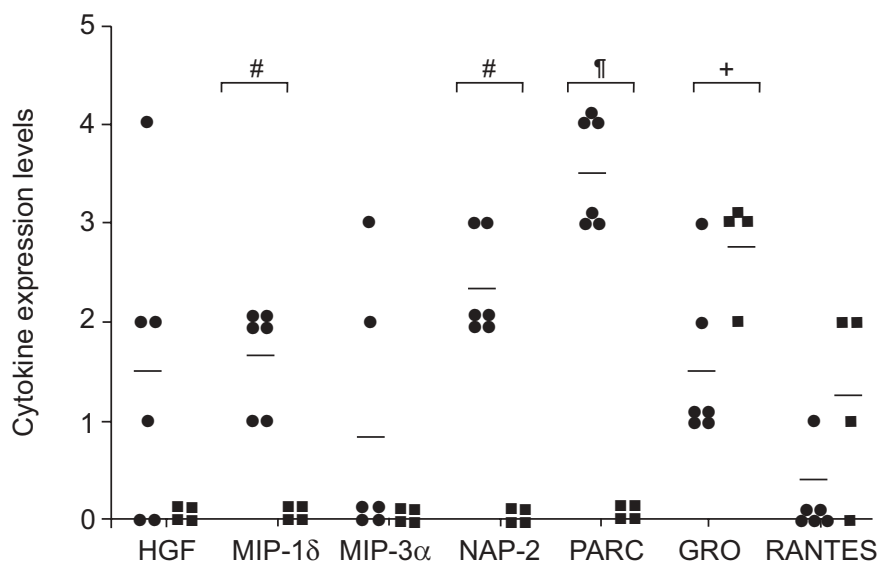

FIGURE 1. Expression levels of cytokines present in effusion $(\bullet)$ and in the supernatant of mesothelioma cell lines ( $\mathbf{0}$ ) assayed by human cytokine antibody arrays. The relative expression levels were determined by density scoring as described in the Methods section. Horizontal bars represent mean values. HGF: hepatocyte growth factor; MIP: macrophage inflammatory protein; NAP: neutrophilactivating peptide; PARC: pulmonary and activation-regulated chemokine; GRO: growth-related oncogene; RANTES: regulated on activation, normal T-cell expressed and secreted. ${ }^{*}: p=0.006 ;{ }^{\bullet}: p=0.007 ;{ }^{+}: p=0.040$.

\section{Effects of in vivo CD4+CD25+ T-cell depletion on tumour growth}

The mesothelioma mouse model described in a previous study [2] was used to examine the impact of CD4+CD25+ T-cell depletion on tumour progression. Preliminary studies indicated that in BALB/c mice, CD25 expression was restricted to CD4+ T-cells and represented a small fraction of total cells in the blood, lymph nodes and spleen $(2-10 \%)$. Kinetic studies have shown that injection of the depleting antibody led to the selective loss of CD4+CD25+ T-cells for $\geqslant 30$ days and that replenishment of the population was observed 50 days after treatment [8]. The present authors first investigated the effect of in vivo administration of anti-CD25 ascites fluid on the CD25+ population in blood, as revealed by flow cytometry. After a single i.p. injection of $0.5 \mathrm{~mL}$ PC61 ascites fluid, this population of cells decreased in blood from 3.5 to $1 \%$ after 25 days (fig. 5).

In this protocol, BALB/c mice were injected i.p. with PBS or PC61 ascites fluid 25 days before tumour cell inoculation. On day 0 , all mice were injected i.p. with a lethal dose of $0.5 \times 10^{6}$ AB1 tumour cells. First signs of terminal illness (typically formation of ascites, ruffled hair or marked loss of condition) appeared after 6 days in both groups (fig. 6). Mice were subjected to extensive autopsy that always showed solid tumour formation within the peritoneal cavity, accompanied in a few cases by thick, yellow-stained ascites. The nature of the solid tumours varied from numerous small nodules spreading throughout the mesentery and peritoneal lining to a single large mass. Within 40 days, all mice from the PBS group showed evidence of ill health or overt tumour growth. The administration of anti-CD25 antibodies prolonged the median survival from 19 to 33 days. Strikingly, five of the 12 mice $(41 \%)$ treated with depleting antibody PC61 remained tumour-free for 2 months. Mice were then sacrificed and 

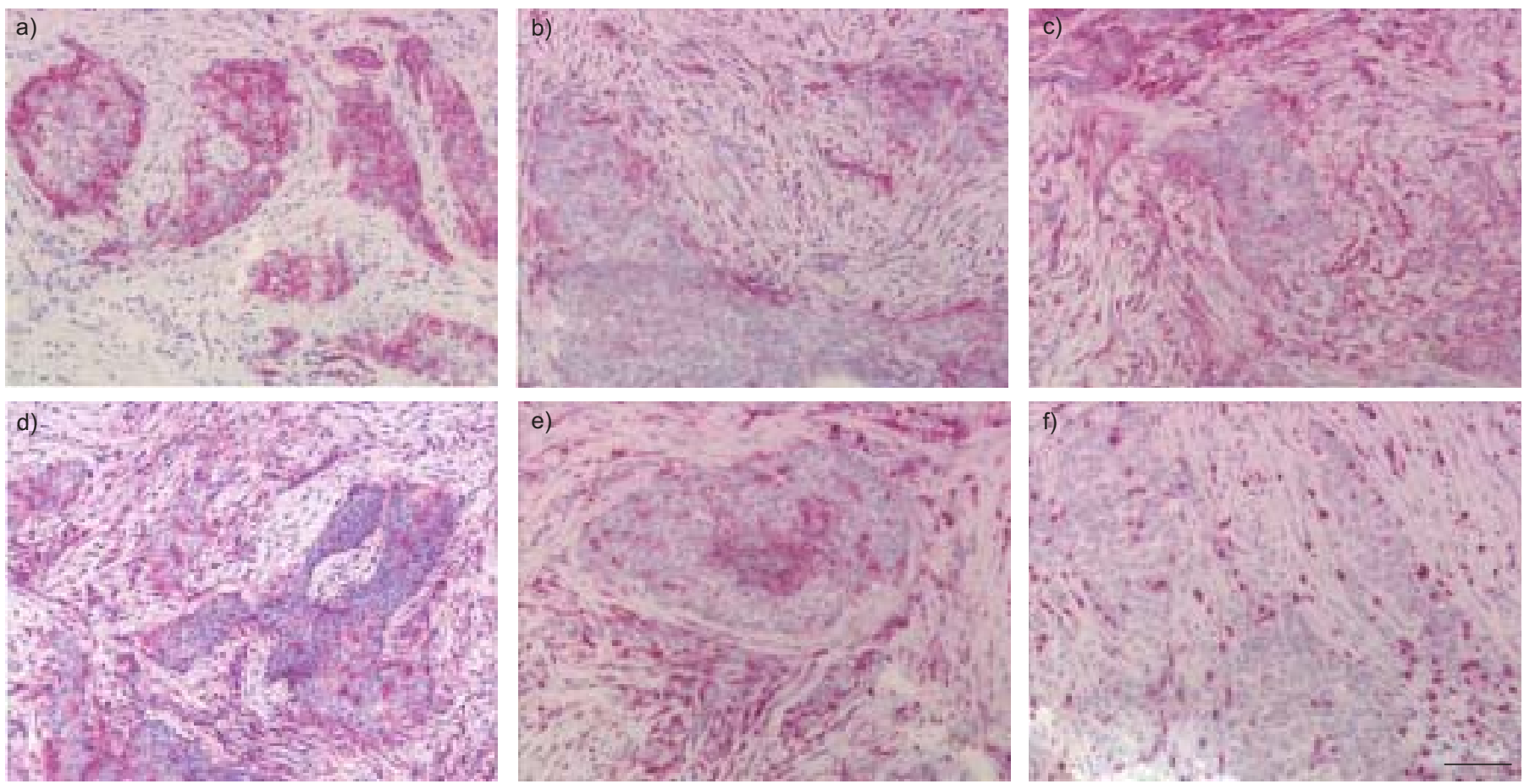

FIGURE 2. Tumour cells were localised in tumour fields as visualised by HBME-1 (a) and cytokeratin staining (RCK108; data not shown). Blood vessels (CD31; b) were present mainly at the periphery of the tumour and are considered a key step in tumour growth. Macrophages (CD68; c), natural killer cells (CD16; d), and T-lymphocytes, both T-helper/inducer (CD4; e) and T-suppressor/cytotoxic (CD8; f) cells, constitute the major part of the inflammatory cell infiltration. Scale bar=100 $\mu \mathrm{m}$.

checked for tumour growth. No tissue abnormalities or formation of tumours could be detected.

\section{DISCUSSION}

Cancer, and in particular mesothelioma, is a chronic disease. When mesothelioma becomes clinically visible $20-40$ yrs after asbestos exposure, tumour cells and their products have already been interacting with and affecting host cells for a considerable time to ensure the survival of the tumour. The paths that mesothelial cells take on their way to becoming malignant is unknown and probably highly variable depending on several host factors, including environmental factors, polymorphisms and mutations in susceptibility genes, age and immunity. Tumour development also depends on factors in the micro-environment. Interactions between malignant cells, stromal cells, extracellular-matrix components, various inflammatory cells, and a range of soluble mediators contribute to tumour development and progression. Mesothelioma tumour of patients with advanced-stage disease is composed of more than just cancer cells; it consists of an intricate network of cell types, including endothelial cells that comprise blood vessels and stromal cells. Many immunological cell types surround and penetrate the cancer cell areas, yet tumours escape immune destruction. There is accumulating evidence that cancer cells can even recruit and subvert normal cell types to serve as active collaborators in their neoplastic programme [9]. Understanding these multiple factors that come into play at the tumour micro-environment level may help to better understand and design immunotherapy protocols. The present authors therefore took an unbiased look at which inflammatory cell types were present inside mesothelioma, and which cytokines and chemokines were produced by mesothelioma cell lines and were present in corresponding pleural fluid. One remarkable observation of the present authors' immunohistological studies was that mesothelioma is heavily infiltrated with many immune effector cells. Macrophages, NK cells, and
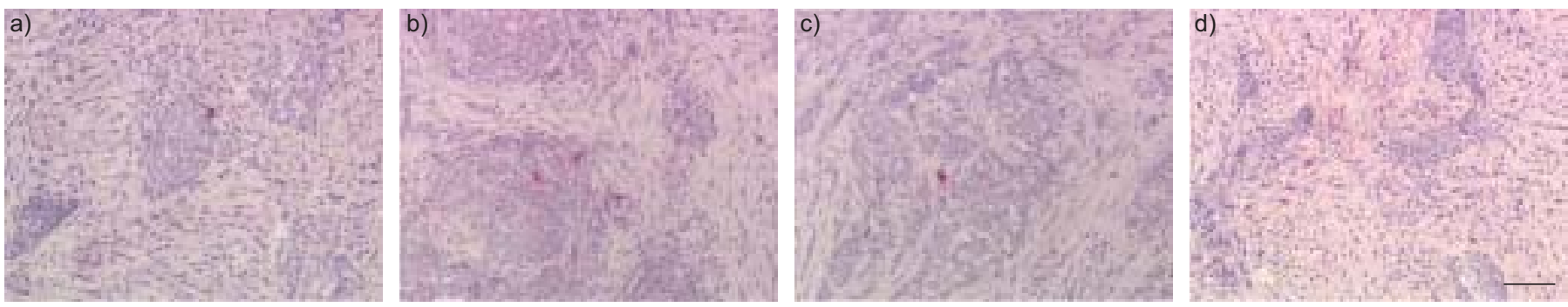

FIGURE 3. Dendritic cells (langerin (a), CD1a (b)), eosinophils (BMK-13; c) and B-cells (CD24; d) were rarely detected. Cells were counter-stained with haematoxylin. Scale bar $=100 \mu \mathrm{m}$ 

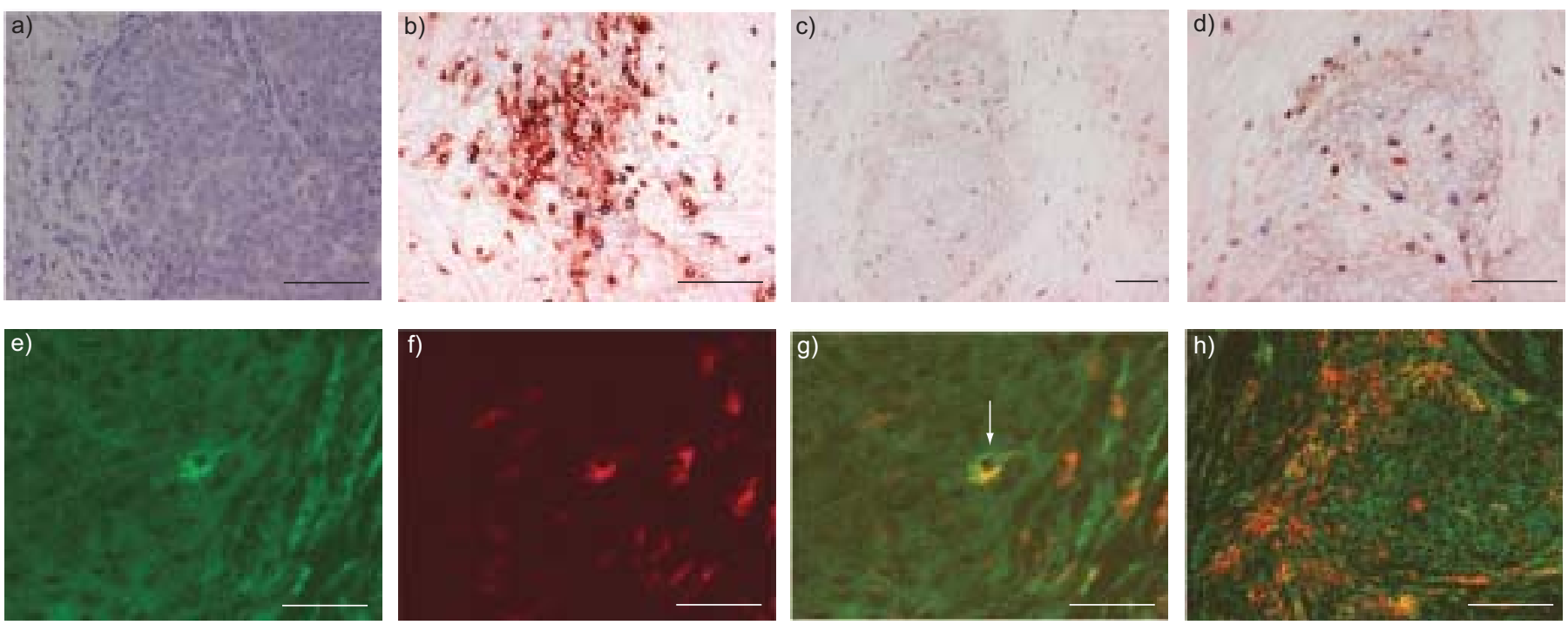

FIGURE 4. a) The transcription factor Foxp3 as a hallmark of naturally arising CD4+CD25+ T-regulatory cells was expressed in the vicinity of the tumour (red staining). Cells were counter-stained with haematoxylin. Expression of Foxp3 (blue staining) strongly correlates with b) CD3 (red staining) or c) and d) CD25 expression (red staining). There was no counter-staining in these cells. Fluorescent microscopy on mesothelioma tissue sections showing e) fluorescein isothiocyanate-labelled CD25+ cells, f) Alexa Fluor 647-labelled anti-human CD4+ cells and g) CD4+CD25+ double positive cells (indicated by arrow). $g$ and h) show the appearance of CD4+CD25+ double positive cells at different magnifications. Scale bars $=100 \mu \mathrm{m}(\mathrm{a}-\mathrm{d}, \mathrm{h})$ and $25 \mu \mathrm{m}(\mathrm{e}-\mathrm{g})$.
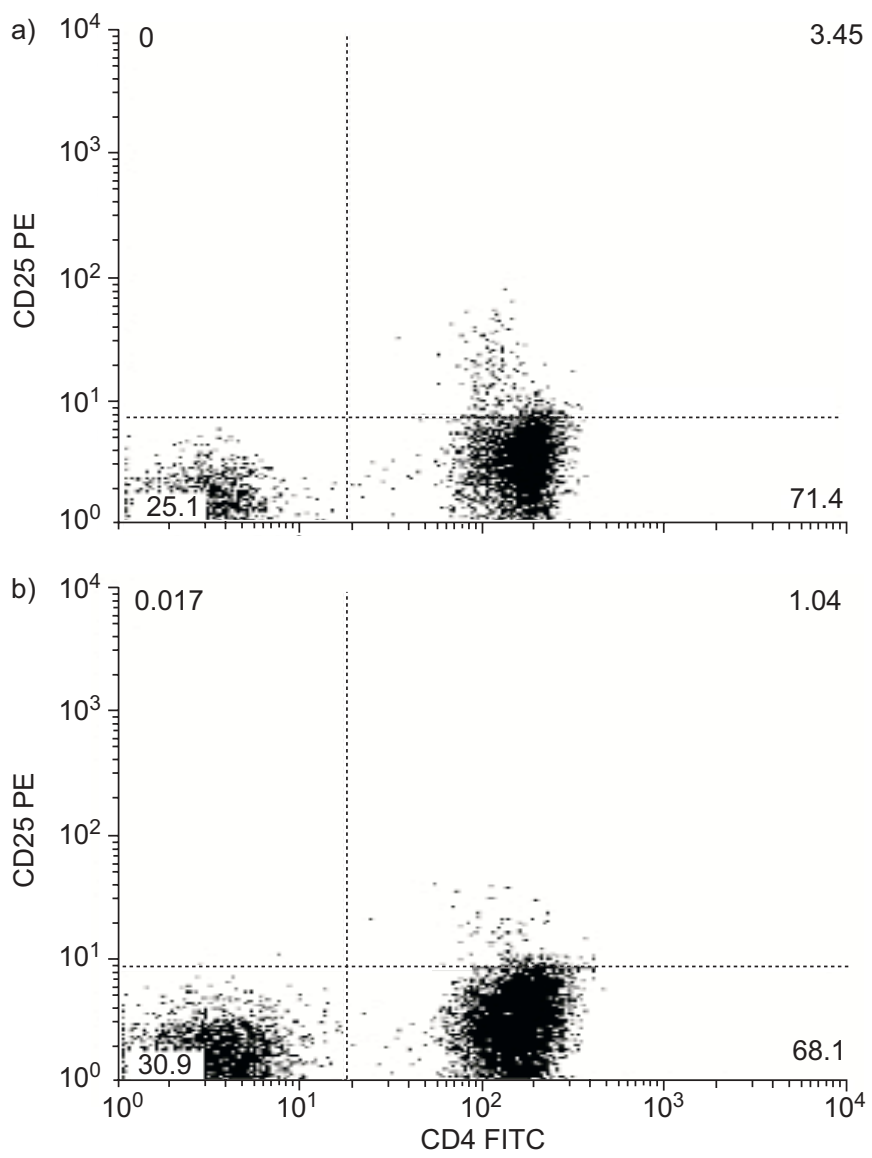

FIGURE 5. Flow cytometry analysis of blood from BALB/c mice that were a) untreated or b) treated with $0.5 \mathrm{~mL}$ PC61 ascites fluid on day 25 after antibody administration. PE: phycoerythrin; FITC: fluorescein isothiocyanate.
T-lymphocytes, both T-helper/inducer (CD4) and T-suppressor/cytotoxic (CD8) cells, constituted the major part of the inflammatory cells. Not surprisingly, the present protein array demonstrated the production of many chemokines in both MM cell line supernatant and pleural fluid (interferon-inducible protein-10, MIF, monocyte chemoattractant protein-1, epithelial neutrophil-activating protein-78, MIP-1 $\beta$, IL-8, GRO, RANTES) or exclusively in pleural fluid (MIP- $1 \delta$, MIP- $3 \alpha$, NAP-2, and PARC) with the potential to attract these cell types.

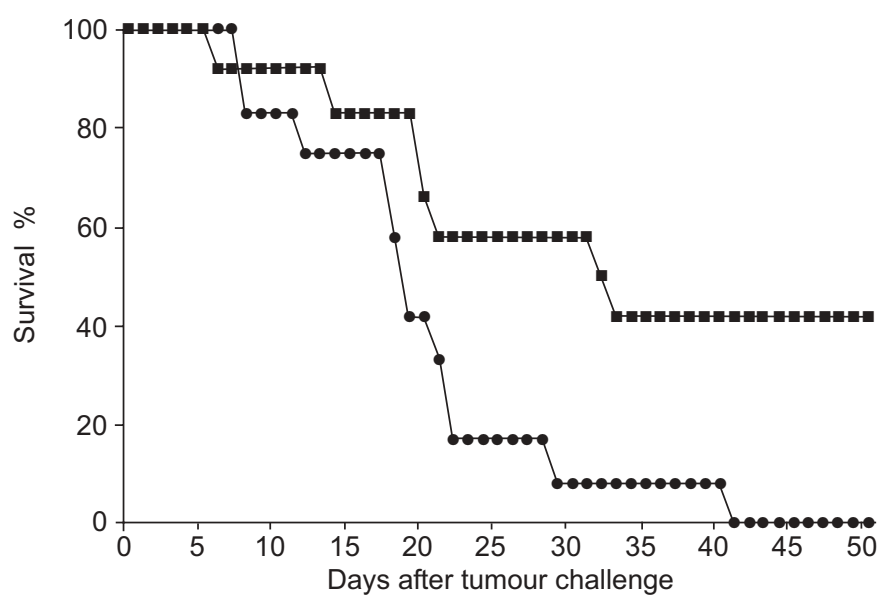

FIGURE 6. Kaplan-Meier survival plot showing the effect of depleting CD4+CD25+ T-cells by the administration of anti-CD25 antibody (PC61) in the development of malignant mesothelioma in a mouse model. Twenty-five days before tumour cell inoculation, mice were injected intraperitoneally with PBS ( $\mathrm{n}=12)$ or depleting antibody PC61 $(\mathbf{\square}, \mathrm{n}=12)$. On day 0 , mice were subjected to a lethal dose of $0.5 \times 10^{6} \mathrm{AB} 1$ tumour cells. Mice were scored when profoundly ill to UK coordinating Committee on Cancer Research regulations and by the Code of Practice of the Dutch Veterinarian Inspection. $\mathrm{p}<0.028$ determined by log-rank test. 
The role of macrophages, T-cells and other immune cells in mesothelioma biopsies is still unknown. The influx, probably caused by changes in the microenvironment of the tumour, can favour inflammation, angiogenesis and tumour growth, thus leading to tumour progression. It can also negatively impact on tumour growth; for example, the presence of macrophages in tumour islets of nonsmall cell lung cancer (NSCLC) and the presence of infiltrating effector memory T-cells in colorectal cancer is associated with an increased survival [10, 11]. Understanding the outcome of the anti-tumoural immune response, leading to tumour progression or regression, and the role of individual cell types on outcome is of major importance for the design of future clinical trials.

Eosinophils, mast cells, B-cells and neutrophils were rarely detected. Mast cell infiltration and their density in tumour islets have been described as predictors of survival in NSCLC [10]. The difference in mast cell density in NSCLC and mesothelioma may be caused by discrepancies in the type, stage and size of the tumours and the methods used to assess these cells. WELSH et al. [10] used anti-tryptase to detect all mast cells, whereas the present authors stained for the chymase+ mast cell. This discrepancy between tryptase and chymase has also been illustrated in malignant breast tumours [12]. Clinical follow-up data comparing chymase+ and tryptase+ in human mesothelioma biopsies in combination with other immune cell markers may reveal more information regarding their prognostic significance.

As with several other cancer types, many immune effector cells were seen within the tumour whilst tumours were still not rejected. One of the aims of the current investigation was to determine the presence of factors within both the mesothelioma and surrounding stromal cells that could suppress the immune response to the tumour. In addition to factors involved in angiogenesis (angiogenin, vascular endothelial growth factor (VEGF), GRO) and leukocyte attraction (chemokines), several factors were discovered that might suppress the anti-tumoural immune response, either by suppressing the antigen-presenting capacity of DCs or the effector arm of the immune system. One of the factors found in pleural fluids of MM patients was HGF, also known as scatter factor. HGF is a multifunctional factor involved both in development and tissue repair, as well as pathological processes such as cancer and metastasis [13]. It was previously shown that HGF has a predominant role in mesothelioma cell invasion, simultaneously stimulating adhesion, motility, invasion and regulation of matrix metalloproteinase and tissue inhibitor of metalloproteinase levels [14]. Strikingly, it was shown that HGF has the potential to induce GRO and VEGF (also found in the present analysis) in a number of tumour models, including NSCLC [15]. HGF might be a critical mediator of immune suppression in $\mathrm{MM}$ as it was recently shown that HGF suppresses the maturation status and antigen-presenting capacity of lung DCs [16]. Similarly, the present authors discovered that VEGF was secreted by MM cell lines and was present in pleural effusions. VEGF's main role is inducing angiogenesis to the benefit of the tumour, but it has long been known to critically suppresses the function of DCs in inducing an anti-tumoural response by keeping these cells in an immature state or inhibiting their differentiation from monocytes [17].
Another striking observation of the present study was that CD1a+ DCs could not be found inside the MM biopsies taken. Additional DC markers (langerin, fascin, S100, BDCA2, and CD209) were applied because of the debate on the use of CD1a $[18,19]$, and confirmed this rare detection of DC in mesothelioma biopsies. This is in contrast to many other tumours, such as breast cancer or NSCLC, in which CD1a+ DCs are found within tumour lesions [20]. One possible explanation for the lack of DCs in MM would be the presence of high levels of IL-6 produced by the MM cells, as shown in the present study. IL- 6 has also been shown by others to be universally expressed in MM cell lines [21]. IL-6 suppresses the development of DCs from CD34+ progenitors and from monocytes in vitro [22]. Studies in multiple myeloma patients have demonstrated that high-level IL-6 is responsible for a lack of circulating DCs in these patients [23]. In mice and humans, IL-6 also keeps DCs in a persistently immature state and promotes the differentiation of macrophages from monocytes [22]. The absence of DCs inside tumours has been shown to affect tumour progression in a number of cancers, including lung cancer [24]. DCs inside tumours might be crucial for activating effector CD4 and CD8 cells to exert their effector function locally inside the tumour [25]. The absence of DCs might partially explain why tumours are not killed despite the presence of effector cells inside the tumour. Consequently, increasing the levels of DC differentiation and growth factor granulocyte macrophage colony-stimulating factor have been used in a number of cancers as a strategy to enhance the antitumoural response [26].

Another explanation for the presence of immune effector cells inside tumours despite a lack of anti-tumoural response, is the presence of naturally occurring Treg cells that suppress the anti-tumoural T-cells. In a mouse model of spontaneous tumour development it was shown that this is one of the predominant ways in which tumours evade immune recognition [4]. Naturally occurring Treg cells play an important role in maintaining immunological balance by suppressing a wide variety of immune responses to self-antigens, infectious agents and tumours [27]. This subset of CD4+ T-cells express high levels of CD25 (IL-2 receptor $\alpha$ chain), are naturally anergic and require stimulation through the $\mathrm{T}$-cell receptor for induction of their cell-mediated suppressive function. The forkhead transcription factor Foxp3 is particularly important in the development of these cells [28]. An increased frequency of Treg cells has been observed in peripheral blood and tissues of patients with cancer [29]. In ovarian carcinoma, high numbers of infiltrating CD4+CD25+Foxp3+ Treg cells were associated with worse prognosis, and these cells directly suppressed infiltrating CD4 and CD8 cell function [30]. Similarly, in the present study the authors have demonstrated that human mesothelioma biopsies harbour significant numbers of CD4+CD25+ T-cells, and that these CD25+ cells also express the Treg transcription factor Foxp3. Others studies have also described an increase in CD4+CD25+ cells in MM pleural effusion [31].

To address the function of these Treg cells, the present authors turned to the murine transplantable mesothelioma AB1 mouse model [2]. In this model, tumour growth was significantly reduced and survival increased when Treg cells were depleted using a CD25-depleting antibody prior to tumour 
implantation. The median survival was prolonged from 19 to 33 days. It was noted that five of the 12 treated mice $(41 \%)$ remained tumour-free for 2 months. This is reminiscent of other experimental tumour models in which Treg depletion using the same antibodies led to increased tumour rejection $[32,33]$. One way in which Treg cells mediate their suppressive function is through signalling of the transforming growth factor (TGF)- $\beta$ receptor of CD8 T-cells [34]. In the present study, TGF- $\beta 2$ could be detected in the pleural fluid and MM supernatant, but it is not currently known whether TGF- $\beta$ is produced by CD4+CD25+ Treg cells. Another suppressive cytokine produced by Treg cells is IL-10. Although the present authors were unable to detect IL-10 in the protein array, a weak staining for IL-10 on immunohistology was observed (data not shown). As well as Treg cells and immunosuppressive cytokines in the tumour micro-environment, there are other possible reasons why the immune response against mesothelioma is generally so ineffective. Low or absent production of specific antigens, crypticity of epitopes or downregulation of MHC expression keeps the immune system ignorant of the tumour. Presentation of antigen without adequate co-stimulation may induce clonal anergy or cause effector cells to develop a non-destructive response or one which leads to cell death. Future experiments in mice will explore the functional significance of these cytokines in Treg-mediated immunosuppression. Currently it can only be speculated why high levels of Treg cells with suppressive function are found inside mesothelioma. The present authors have previously described high levels of heat shock protein-70 in MM-derived tumour fractions [5]. It has shown that self-heat-shock protein-derived peptides have the potential to expand Foxp3+CD4+CD25+ Treg cells [35]. Moreover, high levels of cyclooxygenase 2 and prostaglandin- $\mathrm{E}_{2}$ have been found in mesothelioma, and are correlated with worse prognosis [36]. Tumourderived prostaglandin- $\mathrm{E}_{2}$ specifically induces the Foxp3 gene expression and Treg cell function in human CD4+ CD25- Tcells [37].

One way in which the present data might be employed is in the design of better immunotherapeutic trials for mesothelioma. The present authors have recently shown that immunotherapy using tumour-pulsed dendritic cells is effective in preventing the outgrowth of murine mesothelioma, but that success is limited in mice with larger tumour burden [2]. Inhibiting the immunosuppressive milieu of the tumour by tumour debulking or by blocking vascular endothelial growth factor, interleukin-6 or hepatocyte growth factor activation and/or signalling might be used as an adjunct to dendritic cell immunotherapy. Even more challengingly, the inhibition of T-regulatory function by low-dose cyclophosphamide, specific Toll-like receptor 8 agonists, COX2 inhibition or immunotoxins directed at CD25 might be used in combination with dendritic cell immunotherapy to increase the success rate of mesothelioma tumour eradication. Clearly, pre-clinical studies in mouse malignant mesothelioma models will have to demonstrate the success of such a combined approach before a clinical trial is designed.

\section{ACKNOWLEDGEMENTS}

The authors would like to thank G. Oldenhove (Université Libre de Bruxelles, Brussels, Belgium) for providing PC61.

\section{REFERENCES}

1 Robinson BW, Musk AW, Lake RA. Malignant mesothelioma. Lancet 2005; 366: 397-408.

2 Hegmans JP, Hemmes A, Aerts JG, Hoogsteden HC, Lambrecht $\mathrm{BN}$. Immunotherapy of murine malignant mesothelioma using rumor lysate-pulsed dendritic cells. Am J Respir Crit Care Med 2005; 171: 1168-1177.

3 Dunn GP, Bruce AT, Ikeda H, Old LJ, Schreiber RD. Cancer immunoediting: from immunosurveillance to tumor escape. Nat Immunol 2002; 3: 991-998.

4 Willimsky G, Blankenstein T. Sporadic immunogenic tumours avoid destruction by inducing T-cell tolerance. Nature 2005; 437: 141-146.

5 Hegmans JP, Bard MP, Hemmes A, et al. Proteomic analysis of exosomes secreted by human mesothelioma cells. Am J Pathol 2004; 164: 1807-1815.

6 Lowenthal JW, Corthesy P, Tougne C, Lees R, MacDonald HR, Nabholz M. High and low affinity IL 2 receptors: analysis by IL 2 dissociation rate and reactivity with monoclonal anti-receptor antibody PC61. J Immunol 1985; 135: 3988-3994.

7 Sakaguchi S. The origin of FOXP3-expressing CD4+ regulatory $\mathrm{T}$ cells: thymus or periphery. J Clin Invest 2003; 112: 1310-1312.

8 Oldenhove G, de Heusch M, Urbain-Vansanten G, et al. CD4+ CD25+ regulatory T cells control T helper cell type 1 responses to foreign antigens induced by mature dendritic cells in vivo. J Exp Med 2003; 198: 259-266.

9 Zou W. Immunosuppressive networks in the tumour environment and their therapeutic relevance. Nat Rev Cancer 2005; 5: 263-274.

10 Welsh TJ, Green RH, Richardson D, Waller DA, O'Byrne KJ, Bradding P. Macrophage and mast-cell invasion of tumor cell islets confers a marked survival advantage in non-small-cell lung cancer. J Clin Oncol 2005; 23: 8959-8967.

11 Pages F, Berger A, Camus M, et al. Effector memory T cells, early metastasis, and survival in colorectal cancer. $N$ Engl J Med 2005; 353: 2654-2666.

12 Kankkunen JP, Harvima IT, Naukkarinen A. Quantitative analysis of tryptase and chymase containing mast cells in benign and malignant breast lesions. Int J Cancer 1997; 72: 385-388.

13 Hiscox S, Parr C, Nakamura T, Matsumoto K, Mansel RE, Jiang WG. Inhibition of HGF/SF-induced breast cancer cell motility and invasion by the HGF/SF variant, NK4. Breast Cancer Res Treat 2000; 59: 245-254.

14 Harvey P, Clark IM, Jaurand MC, Warn RM, Edwards DR. Hepatocyte growth factor/scatter factor enhances the invasion of mesothelioma cell lines and the expression of matrix metalloproteinases. Br J Cancer 2000; 83: 1147-1153.

15 Dong G, Lee TL, Yeh NT, Geoghegan J, Van Waes C, Chen Z. Metastatic squamous cell carcinoma cells that overexpress c-Met exhibit enhanced angiogenesis factor expression, scattering and metastasis in response to hepatocyte growth factor. Oncogene 2004; 23: 6199-6208.

16 Okunishi K, Dohi M, Nakagome K, et al. A novel role of hepatocyte growth factor as an immune regulator through suppressing dendritic cell function. J Immunol 2005; 175: $4745-4753$. 
17 Gabrilovich DI, Chen HL, Girgis KR, et al. Production of vascular endothelial growth factor by human tumors inhibits the functional maturation of dendritic cells. Nat Med 1996; 2: 1096-1103.

18 Ulanova M, Tarkowski A, Porcelli SA, Hanson LA. Antigen-specific regulation of CD1 expression in humans. J Clin Immunol 2000; 20: 203-211.

19 Cappello F, Rappa F, Bucchieri F, Zummo G. CD1a: a novel biomarker for Barrett's metaplasia? Lancet Oncol 2003; 4: 497.

20 Hillenbrand EE, Neville AM, Coventry BJ. Immunohistochemical localization of CD1a-positive putative dendritic cells in human breast tumours. Br J Cancer 1999; 79: 940-944.

21 Schmitter D, Lauber B, Fagg B, Stahel RA. Hematopoietic growth factors secreted by seven human pleural mesothelioma cell lines: interleukin-6 production as a common feature. Int J Cancer 1992; 51: 296-301.

22 Chomarat P, Banchereau J, Davoust J, Palucka AK. IL-6 switches the differentiation of monocytes from dendritic cells to macrophages. Nat Immunol 2000; 1: 510-514.

23 Ratta M, Fagnoni F, Curti A, et al. Dendritic cells are functionally defective in multiple myeloma: the role of interleukin-6. Blood 2002; 100: 230-237.

24 Inoshima N, Nakanishi Y, Minami T, et al. The influence of dendritic cell infiltration and vascular endothelial growth factor expression on the prognosis of non-small cell lung cancer. Clin Cancer Res 2002; 8: 3480-3486.

25 Zammit DJ, Cauley LS, Pham QM, Lefrancois L. Dendritic cells maximize the memory CD8 $\mathrm{T}$ cell response to infection. Immunity 2005; 22: 561-570.

26 Davidson JA, Musk AW, Wood BR, et al. Intralesional cytokine therapy in cancer: a pilot study of GM-CSF infusion in mesothelioma. J Immunother 1998; 21: 389-398.

27 Sakaguchi S. Naturally arising CD4+ regulatory t cells for immunologic self-tolerance and negative control of immune responses. Annu Rev Immunol 2004; 22: 531-562.

28 Fontenot JD, Rudensky AY. A well adapted regulatory contrivance: regulatory $\mathrm{T}$ cell development and the forkhead family transcription factor Foxp3. Nat Immunol 2005; 6: 331-337.

29 Wolf AM, Wolf D, Steurer M, Gastl G, Gunsilius E, Grubeck-Loebenstein B. Increase of regulatory $\mathrm{T}$ cells in the peripheral blood of cancer patients. Clin Cancer Res 2003; 9: 606-612.

30 Curiel TJ, Coukos G, Zou L, et al. Specific recruitment of regulatory $\mathrm{T}$ cells in ovarian carcinoma fosters immune privilege and predicts reduced survival. Nat Med 2004; 10: 942-949.

31 DeLong P, Carroll RG, Henry AC, et al. Regulatory T cells and cytokines in malignant pleural effusions secondary to mesothelioma and carcinoma. Cancer Biol Ther 2005; 4: 342-346.

32 Sutmuller RP, van Duivenvoorde LM, van Elsas A, et al. Synergism of cytotoxic T lymphocyte-associated antigen 4 blockade and depletion of CD25(+) regulatory $\mathrm{T}$ cells in antitumor therapy reveals alternative pathways for suppression of autoreactive cytotoxic T lymphocyte responses. J Exp Med 2001; 194: 823-832.

33 Steitz J, Bruck J, Lenz J, Knop J, Tuting T. Depletion of CD25(+) CD4(+) T cells and treatment with tyrosinaserelated protein 2-transduced dendritic cells enhance the interferon alpha-induced, CD8(+) T-cell-dependent immune defense of B16 melanoma. Cancer Res 2001; 61: 8643-8646.

34 Chen ML, Pittet MJ, Gorelik L, et al. Regulatory T cells suppress tumor-specific CD8 T cell cytotoxicity through TGF-beta signals in vivo. Proc Natl Acad Sci USA 2005; 102: 419-424.

35 van Eden W, van der Zee R, Prakken B. Heat-shock proteins induce T-cell regulation of chronic inflammation. Nat Rev Immunol 2005; 5: 318-330.

36 Edwards JG, Faux SP, Plummer SM, et al. Cyclooxygenase2 expression is a novel prognostic factor in malignant mesothelioma. Clin Cancer Res 2002; 8: 1857-1862.

37 Baratelli F, Lin Y, Zhu L, et al. Prostaglandin E2 induces FOXP3 gene expression and $\mathrm{T}$ regulatory cell function in human CD4+ T cells. J Immunol 2005; 175: 1483-1490. 